\title{
Kollaborative Wege in die Smart City
}

\section{Dynamische Planungsinstrumente für die kommunale Wärmewende}

\author{
André Ortiz, InWIS GmbH - Institut für Wohnungswesen, Immobilienwirtschaft, Stadt- und Regionalentwicklung an der EBZ Business School \\ und der Ruhr-Universität Bochum, Springorumallee 20 a, 44795 Bochum (andre.ortiz@inwis.de) (D) https://orcid.org/0000-0002-6737-1864 \\ Dorothea Ludwig, IP SYSCON GmbH (dorothea.ludwig@ipsyscon.de) \\ Frithjof Pollmüller, IP SYSCON GmbH (frithjof.pollmueller@ipsyscon.de) \\ Daniela Becker, IZES gGmbH (dbecker@izes.de) \\ Florian Noll, IZES gGmbH (noll@izes.de)
}

Im Mittelpunkt dieses Beitrags steht ein kollaborativer Ansatz zur Entwicklung eines dynamischen Wärmekatasters, das als Planungsinstrument für die Wärmewende in der Smart City und zur Erreichung des Ziels klimaneutraler Städte dienen soll. Vor diesem Hintergrund wird aufgezeigt, wie in einem transdisziplinären Technikentwicklungsprozess Elemente der Nutzer*innenbeteiligung zum Tragen kommen und wie auf diese Weise ein an die mehrschichtigen Anforderungen der Stakeholder angepasstes Instrumentarium entwickelt werden kann.

\section{Collaborative approaches to the smart city}

Dynamic planning instruments for the municipal heat transition

This article focuses on a collaborative research approach to developing a dynamic heat map as a planning tool for the heat transition in smart cities and for achieving the goal of climate-neutral cities. Against this background, it is shown how elements of user involvement come into play in a transdisciplinary technology development process and how, in this way, a set of instruments can be developed that is adapted to the multi-layered requirements of the stakeholders.

Keywords: heat planning, governance, collaboration, user involvement, smart city

\section{Die Smart City als Kontext der Wärmeplanung}

Die Smart City stellt einen Kulminationspunkt des Einsatzes digitaler Technologien und der Hervorbringung von Lösungsansätzen zu Herausforderungen nachhaltiger Entwicklung im urbanen Raum dar. Lösungsansätze können sich als technische Innovationen für den Stadtraum beziehungsweise als Innovationen der Stadtentwicklung darstellen und umfassen auch organisatorische und politische Elemente sowie die Rolle der Zivilgesellschaft (Löw und Sept 2019, S. 1 und 8 f.). Das Konzept der Smart City umfasst ein breites Spektrum an Handlungsfeldern, von der Infrastruktur für Informations- und Kommunikations-Technologie (IKT), z. B. 5G-Netz, über E-Governance, Mobilität, Energie, Umwelt, Wohnen, Wirtschaft bis hin zu kommunalen Leistungen (Soike und Libbe 2018, S. 11). Im Überlagerungsbereich von Prozessen der Digitalisierung und integrierter Stadtentwicklung bildet die Smart City auch den Betrachtungs- und Gestaltungsrahmen für die Entwicklung von Ansätzen der Energie- und Wärmewende. Diese Ansätze zeichnen sich durch den Zusammenhang politischer, technischer und sozialer Elemente sowie die räumliche Einbettung, Datenbezogenheit und Mannigfaltigkeit konkreter Maßnahmen aus, die speziell auf der kommunalen Ebene verortet werden (Pehnt und Nast 2016).

Ausgehend von der Gestaltung förderlicher regulatorischer Rahmenbedingungen auf der Landesebene beziehen sich Maßnahmen der Wärmewende u. a. auf die Erschließung lokaler Wärmequellen, das Voranbringen von Fernwärmelösungen unter Vermeidung von Doppelstrukturen (Gas und Fernwärme) sowie die sozialverträgliche Umsetzung energetischer Sanierungen. Zunehmende Bedeutung erlangt hierbei die Etablierung von Quartierskonzepten, durch welche Quartiere Keimzellen des gemeinsamen Handelns von öffentlicher Verwaltung und Wohnungsunternehmen bei der Wärmeversorgung ausbilden (Dun-
This is an article distributed under the terms of the Creative Commons Attribution License CCBY 4.0 (https://creativecommons.org/licenses/by/4.0/)

https://doi.org/10.14512/tatup.301.43

Submitted: 18. 09.2020. Peer reviewed. Accepted: 29. 01.2021 
kelberg et al. 2020, S. iii). Speziell in Konzepten und umgesetzten Modellprojekten der Smart City liegt das Augenmerk auf dem Einsatz energieoptimierender Effizienztechnologien in Privathäusern, Bürogebäuden und Hotels in Verbindung mit der Förderung eines bewussten Umgangs mit Strom und Heizenergie (Vogel et al. 2018, S. 48).

Dreh- und Angelpunkt für konkrete Maßnahmen ist die komplexe Aufgabe der Wärmeplanung auf der kommunalen Ebene. Hierbei nehmen Kommunalverwaltungen, Energieversorger, Dienstleister*innen und Wohnungsunternehmen bzw. Immobilieneigentümer*innen die Wärme-Infrastrukturen, Quartierskontexte, Gebäudeparameter sowie das Nutzungsverhalten in den Blick. Im Sinne dieses Beitrags sind mit der Wärmeplanung auch grundlegende kommunikative Aufgaben verbunden (Abb. 1). Seit den 1980er-Jahren werden zunehmend Wärmekataster als ein Instrument der kommunalen Wärmeplanung eingesetzt, in denen basierend auf einem Geoinformationssystem (GIS) raum- und zeitbezogene Daten des Wärmebedarfs bzw. -verbrauchs erfasst, verwaltet, analysiert und visuell (kartografisch) dargestellt werden (Becker et al. 2018, S. 13). Aus der Perspektive soziotechnischer Systeme bildet das Wärmekataster ein (Teil-)System innerhalb der Smart City, das von der Kombination und Wechselwirkung technischer und sozialer Elemente mit einem Fokus auf sozioökonomische Rahmenbedingungen sowie Anpassungs- und Abstimmungsprozesse geprägt ist (Hirsch-Kreinsen 2018, S. 13 f.).

Je nach Planungszweck soll das Wärmekataster unterschiedlichen Stakeholdern als Basis für Entscheidungen zur Umsetzung von energetischen Maßnahmen an Gebäuden, die Identi- schen Diskurs Anhaltspunkte für die Mehrwerte der systematischen Einbeziehung unterschiedlicher Stakeholder-Positionen in die Gestaltung transformativer Prozesse im Zuge der Energiewende (Schmidt-Scheele et al. 2019). Insgesamt bestehen entsprechend hohe Anforderungen an die Verfügbarkeit und Qualität der Datengrundlage für das Wärmekataster, das sich als ein Teil des urbanen Datenraums in die IKT- und Dateninfrastruktur der Smart City einfügt (Schieferdecker et al. 2018, S. 14 ff.).

\section{Dynamisierung von Wärmekatastern durch Kollaboration}

Ein wichtiger neuer Schritt zur Verbesserung der Qualität und Zuverlässigkeit der Datengrundlage von Wärmekatastern ist deren Dynamisierung, d.h. die Erhebung bzw. Aktualisierung und Fortschreibung von gebäudebezogenen Daten, die für die Wärmebilanzierung relevant sind und für die Abschätzung des (Quartiers-)Wärmebedarfs genutzt werden können. Eine wesentliche Voraussetzung für und ein Mittel zur Dynamisierung ist Kollaboration, in diesem Zusammenhang verstanden als die integrative Vernetzung von relevanten Stakeholdern in Wärmeplanungsprozessen und ihre entsprechende Einbeziehung in den Entwicklungsprozess des dynamischen Wärmekatasters sowie in Ansätze zur Datengewinnung. In diesem Sinne wird im Rahmen des Forschungsprojekts DynamiKol eine GIS-basierte Software-Anwendung konzipiert und prototypisch entwickelt, die als kollaboratives Planungsinstrument in kommunalen Wärmewendeprozessen

\section{Die frühe Einbindung von Nutzer*innengruppen \\ stellt einen innovativen Ansatz für das Forschungs- und Entwicklungsfeld der Wärmeplanung dar.}

fikation von Eignungsgebieten für Wärmenetze, die Ermittlung von Sanierungspotenzialen oder das Monitoring von Sanierungsraten und -tiefen dienen. Abhängig von den Voraussetzungen in den Kommunen besteht eine große Herausforderung jedoch darin, aus vorhandenen aber für die Wärmeplanung unzureichenden zentralen (z. B. das amtliche Liegenschaftskatasterinformationssystem $A L K I S^{\circledR}$ oder die Bevölkerungs-, Gebäude- und Wohnungszählung Zensus) sowie dezentralen Datenquellen zunächst ein Wärmeplanungs-Konzept zu entwickeln und eine kohärente Datenbasis aufzubauen (Dunkelberg et al. 2020, S. 7 f.). Internationale und zunehmende nationale Erfahrungen sprechen dafür, dass regionale bzw. lokale Kenntnisse und Beiträge entscheidend sind für eine erfolgreiche Wärmeplanung, die sich auf Engagement, Zustimmung und Informationen der Stakeholder vor Ort stützen sollte (Ea Energy Analyses und Viegand and Maagøe 2019, S. 6). Schließlich mehren sich auch im methodi- einsetzbar ist. Relevante Stakeholder der Wärmeplanung werden in ihrer Rolle als zukünftige Nutzer*innen über den gesamten Entwicklungsprozess hinweg in das Projekt eingebunden.

Kollaboration in Form von Nutzer*innenbeteiligung im Technikentwicklungsprozess lässt sich hier als Ausprägung kooperativer Governance verstehen, die speziell im urbanen, kommunalen bzw. regionalen Kontext immer häufiger der Entwicklung von Klimaschutzkonzepten und Energieversorgungsstrategien zugrunde liegt (Kropp 2017, S. 140; Sedlacek et al. 2020). Die Einführung solcher kollaborativen Elemente muss eng mit der Weiterentwicklung der technischen Basis des Wärmekatasters zusammengedacht und in einem kohärenten Konzept integriert werden. Es handelt sich insofern um ein inter- und transdisziplinäres Vorhaben der Technikentwicklung, bei dem der informationstechnische Entwicklungsprozess eng an sozialwissenschaftlich flankierte Prozesse der Nutzer*innenbeteiligung 


\begin{tabular}{|c|c|}
\hline Dimension & Leitfragen \\
\hline Methodologie & $\begin{array}{l}\text { Welche Methoden erweisen sich als effektiv, um den kollaborativen Ansatz der Nutzer*innenbeteiligung in den Technikentwicklungs- } \\
\text { prozess zu integrieren? }\end{array}$ \\
\hline Stakeholder/-Interessen & $\begin{array}{l}\text { Wer sind die relevanten Stakeholder innerhalb der Smart City in Bezug auf den Technikentwicklungsprozess und die (integrierte) } \\
\text { Wärmeplanung? }\end{array}$ \\
\hline Ziele/Erwartungen & $\begin{array}{l}\text { Welche Kooperationsebenen (politisch, strategisch, operativ, projektbezogen) und Aktivitäten werden durch Elemente der } \\
\text { Nutzer*innenbeteiligung im Technikentwicklungsprozess adressiert und welche Erwartungen (Anwendungen, Effekte, Mehrwerte) } \\
\text { seitens der Zielgruppen sind damit verbunden? }\end{array}$ \\
\hline Herausforderungen & Welche Hürden, Hemmnisse und Konflikte treten hinsichtlich der Nutzer*innenbeteiligung im Technikentwicklungsprozess zutage? \\
\hline $\begin{array}{l}\text { Implikationen der } \\
\text { Nutzer*innenbeteiligung }\end{array}$ & Wie wirkt sich die Nutzer*innenbeteiligung auf den Technikentwicklungsprozess und die spätere Anwendung der Technik aus? \\
\hline
\end{tabular}

Tab.1: Leitfragen zum kollaborativen Technikentwicklungsprozess bei DynamiKol.

Quelle: eigene Darstellung

gekoppelt ist. Leitfragen des Vorhabens in verschiedenen Dimensionen sind in Tab. 1 zusammengefasst.

Ziel dieses Beitrags ist es, die besondere Rolle der Nutzer*innenbeteiligung im Zuge der technischen Weiterentwicklung des Wärmekatasters als dynamisches Software-Tool und kollaboratives Instrument der kommunalen Wärmeplanung zu beleuchten. Zu diesem Zweck wird vor dem Hintergrund der dargestellten Anforderungen der Wärmeplanung und Leitfragen des Projekts aufgezeigt, welche methodologischen Implikationen sich für die Nutzer*innenbeteiligung im Technikentwicklungsprozess ergeben. Anhand der kommunalen Anwendungsfälle Stadt Essen und Landkreis Gießen wird verdeutlicht, wie in diesem Zusammenhang strukturelle Voraussetzungen, Herausforderungen sowie Erwartungen der Stakeholder zum Tragen kommen.

\section{Nutzer*innenbeteiligung im Technik- entwicklungsprozess}

Im Kern bestehen die Herausforderungen der Wärmekartierung darin, die für Entscheidungen im Rahmen kommunaler Wärmeplanungsprozesse erforderlichen Informationen im Hinblick auf die jeweilige Planungsebene (transsektoral) und Akteurseinbindung (transdisziplinär) auszuwerten, aufzubereiten und zu kommunizieren. Ein innovatives Merkmal des neuen Wärmekatastersystems ist es dementsprechend, immer wieder $(d y$ namisch) auf aktuelle Rahmenbedingungen, Akteurskonstellationen und Datenlagen (z. B. Gebäudesanierung) reagieren zu können und anpassbar zu sein. Jenseits üblicher Auftragsentwicklungsprozesse und ggf. damit verbundener Marktforschung bei ähnlich gelagerten Technologien, erfolgt die Einbindung von Nutzer*innengruppen, u. a. Verwaltungsmitarbeitende, Energieversorger und Wohnungsunternehmen, von der frühen Phase der Konzeptionierung des dynamischen Wärmekatasters an. Dies stellt einen innovativen Ansatz für das Forschungs- und Entwicklungsfeld der Wärmeplanung dar.

Es handelt sich somit um einen Innovationsprozess, bei dem in einer agilen IT-Projektorganisation auch heterogene exter- ne Wissensbestände sowie Wechselwirkungen von Planungsanforderungen im Kontext der Smart City zum Tragen kommen. Um den resultierenden Herausforderungen einer unvollständigen und sich kontinuierlich erweiternden Datenbasis sowie sich im Entwicklungsprozess ändernden Anforderungen gerecht zu werden, liegt der Fokus bei der technischen Konzeption und Entwicklung auf der Flexibilität der eingesetzten Technologien und Methoden. Es wird ein agiles Entwicklungsschema auf Grundlage von sogenannten Usecase-Beschreibungen verfolgt, in dem die Funktionsweisen der einzelnen Systemkomponenten aus Nutzer*innensicht beschrieben und damit eine Abstraktion von der technischen Umsetzungsebene ermöglicht wird. Ziel ist es, mit minimalem Entwurfsaufwand bereits ein lauffähiges System zu schaffen, und es unter Einbeziehung der relevanten Stakeholder durch Anpassung der Usecase-Beschreibungen, iterativ und inkrementell zu erweitern und zu verbessern. Dieses pragmatische Vorgehen beugt Fehlplanungen im späteren Projektverlauf vor, da die zielgerichtete Funktionalität des GISbasierten Wärmekatastersystems regelmäßig geprüft werden kann.

Die Einbindung der Stakeholder erfolgt in verschiedenen Entwicklungsphasen (u. a. Konzeptions-, Test- und Evaluationsphase) gegenstandsangemessen und kann dabei informativen, konsultativen oder auch mitgestaltenden Charakter annehmen bzw. Kombinationen daraus. Resultierendes Feedback fließt in den weiteren Entwicklungsprozess ein und bildet einen wichtigen Faktor für die Adaption des Wärmekatasters an die kommunale Praxis und die systemseitige Umsetzung des Kollaborationsansatzes. Dies erzeugt eine Prozessdynamik, durch die sich Forschungs- und Entwicklungsfragen im Projektverlauf verändern können und in der fortlaufend Ergebnisse und Erkenntnisse Einfluss auf die Gestaltung des weiteren Vorgehens nehmen. Als zentral identifizierte, kommunale Stakeholder-Gruppen, wie Verwaltungsmitarbeitende, Energieversorger und Wohnungsunternehmen, sind in DynamiKol ab der Konzeptionierungsphase beteiligt. Auch in der Entwicklungsphase bleiben sie aktiv eingebunden und tragen $u$. a. Realdaten bei. Eine Ausweitung des Kreises der einzubeziehenden Stakeholder fin- 


\begin{tabular}{|c|c|c|}
\hline Merkmale & Stadt Essen & Landkreis Gießen \\
\hline Raumgliederung & Kreisfreie Großstadt in städtischer Region & Städtischer Kreis in ländlicher Region \\
\hline Auszeichnungen & Grüne Hauptstadt Europas 2017 & Masterplan $100 \%$ Klimaschutz Landkreis \\
\hline Einwohner und Fläche & $583.400 \mathrm{Ew} . / 210,3 \mathrm{~km}{ }^{2}$ & $267.100 \mathrm{Ew} . / 854,7 \mathrm{~km}{ }^{2}$ \\
\hline Bevölkerungsdichte & $2.774 \mathrm{EW} . / \mathrm{km}^{2}$ & $312 \mathrm{Ew} . / \mathrm{km}^{2}$ \\
\hline Flächenverbrauch & $67,1 \%$ & $18,5 \%$ \\
\hline $\begin{array}{l}\text { Durchschnittliches Haushalts- } \\
\text { einkommen (netto) }\end{array}$ & $1.693 €$ & $1.737 €$ \\
\hline $\begin{array}{l}\text { Wohngebäude im Eigentum } \\
\text { von Privatpersonen }\end{array}$ & $67,6 \%$ & $87,1 \%$ \\
\hline Stakeholder-Konfiguration & $\begin{array}{l}\text { Grüne Hauptstadt Agentur, Amt für Stadterneuerung und } \\
\text { Bodenmanagement, Amt für Stadtplanung, Amt für Geo- } \\
\text { information, Stadtwerke, Energieversorger, Wohnungs- und } \\
\text { Immobilienwirtschaft, Wirtschaftsentwicklung, Forschung, } \\
\text { Handwerk, Bürger*innen }\end{array}$ & $\begin{array}{l}\text { Stabstelle Klimaschutz, Masterplanmanager, Stadtwerke, } \\
\text { Energieberatungsnetzwerk, Gemeindevertreter*innen, } \\
\text { Handwerk, Bürger*innen }\end{array}$ \\
\hline $\begin{array}{l}\text { Ansatzpunkte im Wärme- } \\
\text { bzw. Energiesektor }\end{array}$ & $\begin{array}{l}\text { Energienutzungsplan, Klimaschutz-Teilkonzept Wärme, } \\
\text { Ziel der Klimaneutralität bis 2030, Aufbau Kompetenzteam } \\
\text { Energie \& Gebäude, Quartierskonzepte }\end{array}$ & $\begin{array}{l}\text { Wärmesteckbriefe auf Ortsteil-Ebene, Masterplan, Energie- } \\
\text { scouts, Energieberatungsnetzwerk }\end{array}$ \\
\hline
\end{tabular}

Tab.2: Strukturelle Merkmale der kommunalen Beispiele Essen und Gießen.

det in den weiteren Projektschritten der Testung und Evaluation des zu entwickelnden Prototyps entsprechend der antizipierten Nutzer*innengruppen sukzessive statt (Abb. 2). Methodologisch kommen hierzu insbesondere qualitative Ansätze zur Anwendung. Die entsprechenden Methoden und Beteiligungsformate unter Einbezug heterogener Akteure und Wissensbestände ermöglichen eine dichte Beschreibung von Stakeholder-Konstellationen sowie eine differenzierte und diskursive Ermittlung von Akteursanforderungen.

\section{Methodisches Vorgehen in der Konzeptionierungs- phase}

Ein halbtägiger Auftakt-Dialog des transdisziplinären Forschungsvorhabens mit Vertreter*innen der Kommunen Stadt Essen und Landkreis Gießen, die im Projekt als assoziierte Partner fungieren, wurde methodisch als moderiertes Fokusgruppengespräch gestaltet. Den Ausgangspunkt bildeten ein gemeinsamer Problemaufriss und die grundlegende Thematisierung relevanter Aspekte zum projektbezogenen Verständnis kommunaler Wärmeplanung. Hierauf aufbauend wurde die Konzeptidee eines kollaborativen Wärmekatasters anhand der Beschreibung möglicher Funktionalitäten und Nutzer*innengruppen, der wahrgenommenen kommunalen Akteurskonstellationen sowie der relevanten Datenebene des Systems diskutiert. Wesentliche Ergebnisse flossen nach einer Systematisierung und grafischen Aufbereitung in das Ergebnismodell der Konzeptionsphase ein (Abb. 1). Zudem wurden leitfadengestützte Akteursinterviews durchgeführt, bei denen Fragen der Einordnung relevanter Handlungsfelder, Akteurskonstellationen sowie lokaler Spe- zifika in der kommunalen Wärmeplanung im Vordergrund standen. ${ }^{1}$ Die Auswahl der Interviewpartner*innen erfolgte zum einen auf Vorschlag der Ansprechpartner*innen der kooperierenden Kommunen, zum anderen basierend auf konzeptionellen Vorarbeiten zu relevanten Akteursgruppen der kommunalen Wärmeplanung. Weiterhin dienten die Interviews selbst als Quelle der weiteren Stichprobenauswahl (Snowball Sampling).

Mittels einer qualitativen Inhaltsanalyse flossen die Interviewergebnisse hinsichtlich Anforderungsaspekten (Akteursperspektive) in die Erstellung eines kriterienbasierten Anforderungsprofils für ein kollaboratives Wärmekataster ein. Dieses Profil umfasst zum einen Spezifikationen auf der Raum-, Handlungsfeld-, Anwender-, Kollaborations-, Anwendungsfall-, Daten- und Infrastrukturebene. Zum anderen sind darin die Gesichtspunkte Nutzen, Mehrwerte und Hemmnisse jeweils aus der technischen und akteursbezogenen Perspektive sowie in Bezug auf die Skalierbarkeit und Übertragbarkeit berücksichtigt. Hierauf aufbauend wurde eine technische Anforderungsliste erstellt, aus der mögliche Anwendungsfälle bzw. -module eines kollaborativen Wärmekatasters abgeleitet und schließlich eine Grobkonzeption möglicher Anwendungsmodule erstellt wurde.

Zum Ende der Konzeptionierungsphase wurden die erarbeiteten Moduloptionen in Anlehnung an eine multikriterielle Entscheidungsanalyse hinsichtlich ihrer Relevanz für das weitere

1 Im Landkreis Gießen wurden Interviews mit vier Expert*innen geführt: Vertreter*innen kommunaler Verwaltungseinheiten, Energieversorger, Sprecher ${ }^{*}$ in Energieberatungsnetzwerk. In der Stadt Essen wurden mit neun Expert*innen aus sieben organisationalen Kontexten Interviews geführt: Vertreter*innen kommunaler Verwaltungseinheiten, Energieversorger, Wohnungswirtschaft. 


\begin{tabular}{|l|l|}
\hline Entwicklungsmodul & $\begin{array}{l}\text { Funktion und Ziel } \\
\text { Dateneingabe }\end{array}$ \\
\hline Qualitätsverbesserung der Grundlagendaten durch Eingabe realer gebäudebezogener Daten und dadurch Verbesserung \\
\hline Quartiersabgrenzung & Bildung von Quartieren als vorgelagerter Schritt der Datenaufbereitung und räumlicher Bezugspunkt für nachgelagerte Analysen \\
\hline Hochrechnungsalgorithmus & $\begin{array}{l}\text { Statistische Übertragung der über die Dateneingabe erfassten Daten auf den übrigen Gebäudebestand zur Abschätzung } \\
\text { des realen Gesamtwärmebedarfs }\end{array}$ \\
\hline Szenarienrechner & $\begin{array}{l}\text { Darstellung zukünftiger energetischer Entwicklungsoptionen auf Quartiersebene, z. B. als eine Grundlage zur Bewertung } \\
\text { der Resilienz von Städten }\end{array}$ \\
\hline Austauschplattform & Vernetzung der lokalen Akteure der Wärmewende durch die Darstellung von Umsetzungsbeispielen, Kontaktbörse und Marktplatz \\
\hline Monitor & $\begin{array}{l}\text { Visualisierung ausgewählter Indikatoren (auch zeitlicher Entwicklungen) zwecks Information, Fortschrittskontrolle/Monitoring } \\
\text { und Bilanzierung }\end{array}$ \\
\hline
\end{tabular}

Tab.3: Ziele der im Vorhaben DynamiKol ausgewählten Module.

Quelle: eigene Darstellung

Vorhaben anhand von Kriterien der technischen Machbarkeit, des Innovationsgehalts, der Vereinbarkeit mit den Projektzielen sowie des Umsetzungsaufwands bewertet. Auf dieser Grundlage wurden Prioritäten für die weitere Umsetzung von Anwendungsmodulen gesetzt. Flankiert wurde dieser Konzeptionierungs- und Auswahlprozess durch ähnlich einem Fokusgruppengespräch ausgestaltete Online-Foren mit lokalen Stakeholdern aus den kooperierenden Kommunen ${ }^{2}$, um die erarbeiteten Anforderungen an Anwendungsmodule des kollaborativen Wärmekatasters zu schärfen, mögliche Hemmnisse zu identifizieren, die Anwendungsmodulauswahl zu validieren und Entwicklungspfade gemeinsam zu diskutieren. Im Zuge der Konzeptionierungsphase fand so auch eine Ansprache und Aktivierung weiterer lokaler Akteure für den beteiligungsorientierten Projektverlauf statt.

\section{Konzeption des Wärmekatasters}

\section{Kommunale Anwendungsfälle Essen und Gießen}

Der Technikentwicklungsprozess für das Wärmekataster wird aufbauend auf den Pilot-Anwendungsfällen Stadt Essen und Landkreis Gießen durchgeführt. Ein zentrales Ziel der Fallauswahl besteht im Erreichen einer möglichst großen Übertragbarkeit der Ergebnisse und Anwendbarkeit des Wärmekataster-Tools in verschiedenen kommunalen Kontexten. Die zweckgerichtete Fallauswahl (Purposeful Sampling) von Essen und Gießen bildet zwei unterschiedliche Kommunen mit urban bzw. ländlich geprägten Strukturen ab. In Tab. 2 sind wesentliche Strukturmerkmale sowie Stakeholder-Konfigurationen und bestehende Ansatzpunkte der Energie- und Wärmeplanung in den beiden Kommunen zusammengefasst. Die Fallauswahl ermög-

2 Am Online-Forum für den Landkreis Gießen nahmen fünf Personen aus drei Institutionen teil: kommunale Verwaltungseinheiten, Energieversorger, Sprecher*in Energieberatungsnetzwerk. Am Online-Forum für die Stadt Essen nahmen fünf Personen aus vier Institutionen teil: kommunale Verwaltungseinheiten, Energieversorger, Wohnungswirtschaft. licht die Identifikation übergreifender oder spezifischer Anforderungsprofile. Ergebnis der Prüfung von Bevölkerungsstrukturdaten im Rahmen der Fallauswahl ist zudem, dass Testgebiete in den Kommunen möglichst repräsentativ für die Strukturen auf Bundesebene sind.

\section{Technische Konzeptionierung kommunaler Anforderungen}

Im Rückbezug auf die Leitfragen sind die Ergebnisse aus den Beteiligungsformaten und Interviews Grundlage für die technische Konzeptionierung möglicher Anwendungsmodule. Ausgehend von den bereits bestehenden Basisfunktionalitäten eines dynamischen Wärmekatasters und dem Kernelement einer geteilten Datenbasis, legen die Ergebnisse insbesondere drei Entwicklungsdimensionen sowie einen modularen Aufbau des Wärmekatastersystems nahe: Neben der Erweiterung der Grundfunktionalitäten und zusätzlicher Anwendungsmodule der Systembasis zeigt sich dies in der Konzeptionierung damit verknüpfter, möglicher Anwendungsmodule mit Blick auf die fachplanerische Ebene sowie insbesondere hinsichtlich der Integration von Anwendungsmodulen, die auf Kommunikationsprozesse zwischen den heterogenen Akteurskonstellationen im Bereich der kommunalen Wärmeplanung referieren.

Die so konzeptionierten Module sind in Abb. 1 den drei Bereichen Basis, Planung und Kommunikation zugeordnet und mit den potenziellen Nutzer*innengruppen bzw. Stakeholdern verknüpft. Die geteilte Datenbasis steht als verbindendes Element im Zentrum des Modells. Davon ausgewählt als die im Projektrahmen prioritär zu entwickelnden Anwendungen sind die in Abb. 1 mit einem Stern gekennzeichneten Module.

Bei der Auswahl der Bausteine durch das multikriterielle Bewertungsverfahren konnten unterschiedliche, sich zum Teil entgegenstehende Anforderungsdimensionen kommunaler Wärmewendeprozesse berücksichtigt und so eine Abwägung u. a. zwischen Anforderungen der Nutzer*innen, Datenverfügbarkeit, Datenschutz und technischer Umsetzbarkeit ermöglicht werden. 


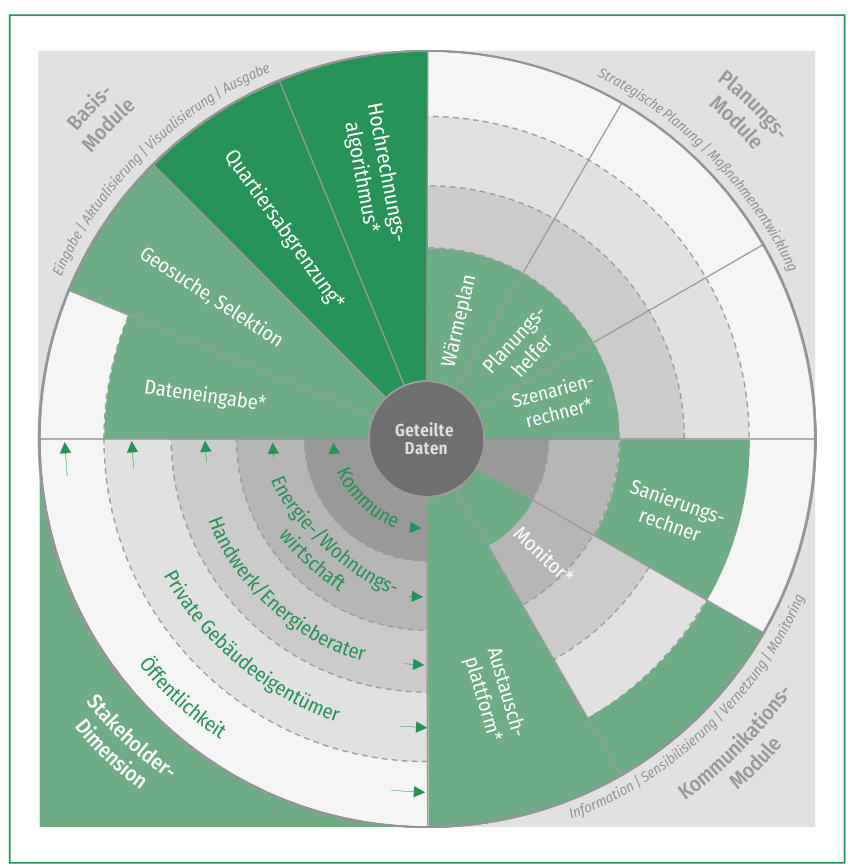

Abb.1: Module des kollaborativen Wärmekatastersystems. Die dunkelgrün hinterlegten Module sind Tools zur Datenvorprozessierung. Es handelt sich bei ihnen im Gegensatz zu den übrigen, grün hinterlegten Modulen nicht um Tools zur regelmäßigen Anwendung durch die Nutzer*innen. Die mit Stern gekennzeichneten Module sollen im weiteren Entwicklungsprozess programmierseitig umgesetzt und getestet werden. Quelle: eigene Darstellung

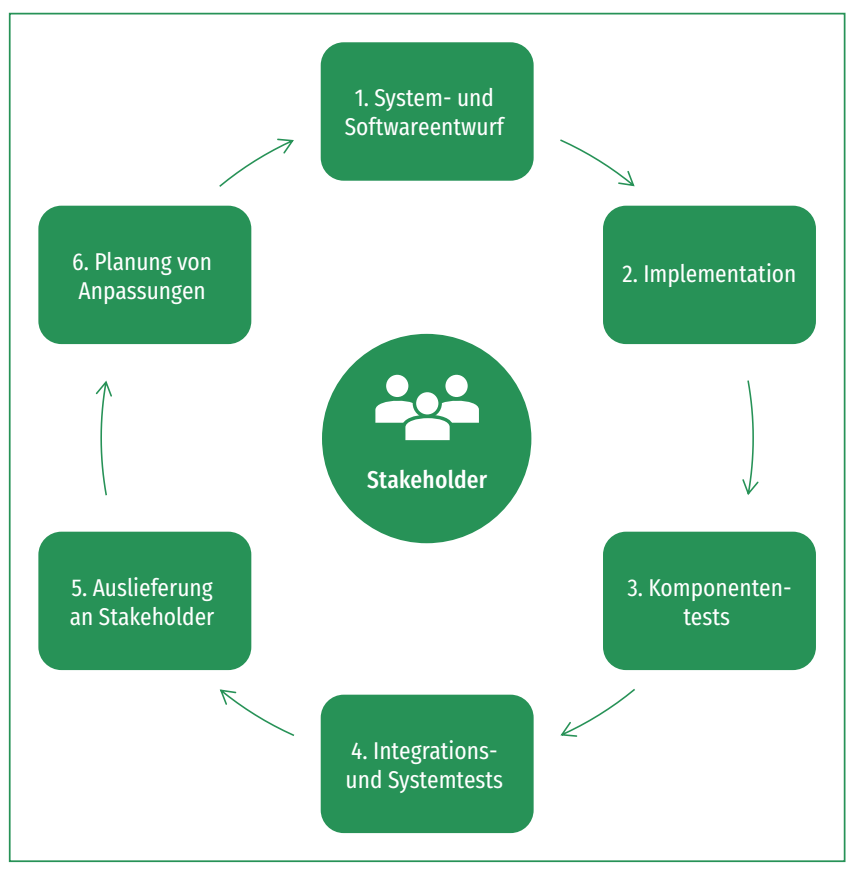

Abb.2: Ausblick auf die idealtypisch dargestellten weiteren Schritte der technischen Entwicklung des Wärmekatasters im Projekt DynamiKol.

Quelle: eigene Darstellung
Dies spiegelt sich in der konzentrischen Zuordnung der Nutzer*innengruppen (Stakeholder-Dimension) in Bezug auf die Nutzung verschiedener Module wider. Funktionsseitig kommt dies z. B. durch einfache Bedienbarkeit und niedrigschwelligen Zugang einerseits sowie detaillierte Einstell- und Eingabemöglichkeiten andererseits zum Ausdruck. Im Ergebnis ergibt sich eine Modulauswahl, durch welche die Stakeholder auf der für sie relevanten Anforderungsebene (Information, Vernetzung, strategische Planung usw.) in den Wärmeplanungsprozess integriert werden.

Die mit den Modulen verfolgten Ziele sind in Tab. 3 zusammengefasst und bilden den Anknüpfungspunkt zur differenzierten Ausarbeitung technischer Usecases im weiteren Forschungsund Entwicklungsprozess für das Wärmekataster.

\section{Fazit und Ausblick}

Zur Realisierung der Wärmewende bedarf es effektiver Instrumente für die Wärmeplanung auf kommunaler Ebene. Ein hierfür geeignetes dynamisches Wärmekataster sollte auf den soziotechnischen Kontext der Smart City ausgerichtet sein und bereits in der Phase seiner Entwicklung die Anforderungen der zentralen Stakeholder einbeziehen. Dieser Beitrag zeigt, wie dies mit einem kollaborativen Ansatz gelingen kann, der die späteren Nutzer*innen von Beginn an in den Entwicklungsprozess einbezieht. Entscheidend ist die Nutzer*innenbeteiligung im Forschungs- und Entwicklungsprozess und ihre reflektierte Umsetzung mithilfe sozialwissenschaftlicher Methoden.

Im Entwicklungsprozess mit den Pilot-Anwendungsfällen Essen und Gießen konnten auf diese Weise in der Konzeptionierungsphase bereits erfolgreich wesentliche Eigenschaften der Anwendung abgestimmt sowie Entscheidungen zur Modulauswahl unter Berücksichtigung der Perspektiven der Stakeholder getroffen werden. Eine abschließende Bewertung, inwieweit sich die Nutzer*innenbeteiligung im Technikentwicklungsprozess auf das Ergebnis auswirkt, ist erst nach Abschluss der weiteren Umsetzung der konzeptualisierten Module möglich. Eine Fortsetzung des beteiligungsorientierten Vorgehens bei der Entwicklung des Wärmekatasters erfolgt in den nächsten Schritten der technischen Umsetzung und Testung, auch unter Einbeziehung weiterer Stakeholder-Perspektiven (insbesondere Handwerk, Bürger*innen) (Abb. 2). Darüber hinaus erscheinen kollaborative Ansätze in Verbindung mit Nutzer*innenbeteiligung als Schlüssel, um auch über den Wärme- und Energiebereich hinaus geeignete Technologien und Instrumente für den Einsatz in der Smart City zu entwickeln.

\section{Erklärung zur Forschungsförderung}

Das Forschungsprojekt DynamiKol („Dynamisierung von Wärmekatastern durch Kollaboration") wird von den Verbundpartnern IZES gGmbH, IP SYSCON GmbH und InWIS GmbH durchgeführt. Förderung: EnEff:Wärme - Forschung für energieeffiziente Wärme- und Kältenetze des Bundesministeriums für Wirtschaft und Energie (BMWi) (Förderkennzeichen: 03ET1620). 


\section{Literatur}

BBSR - Bundesinstitut für Bau-, Stadt- und Raumforschung (2017 a): INKAR. Indikatoren und Daten zur Raum- und Stadtentwicklung. Online verfügbar unter https://www.inkar.de/, zuletzt geprüft am 26.08.2020.

BBSR (2017 b): Raumordnungsregionen 2017. Online verfügbar unter https:// www.bbsr.bund.de/BBSR/DE/forschung/raumbeobachtung/downloads/ downloadsReferenz2.html, zuletzt geprüft am 26.08.2020.

Becker, Daniela; Noll, Florian; Wern, Bernhard; Ludwig, Dorothea; Schröder, Indra (2018): Dynamisierung von Wärmekatastern. Entwicklung und Erprobung technischer Ansätze zur Dynamisierung von kommunalen Wärmekatastern. Online verfügbar unter: http://www.izes.de/sites/default/files/ publikationen/ST_15_063.pdf, zuletzt geprüft am 12.01.2021.

Dunkelberg, Elisa; Weiß, Julika; Hirschl, Bernd (2020): Urbane Wärmewende. Wärmewende in Städten gestalten. Empfehlungen für eine sozial-ökologische Transformation der Wärmeversorgung am Beispiel von Berlin. Berlin: Institut für ökologische Wirtschaftsforschung GmbH.

Ea Energy Analyses; Viegand and Maagфe (2019): Erfahrungen mit der Wärmewende in Dänemark. Input zur Entwicklung eines Leitfadens zur Wärmeplanung in Baden-Württemberg. Online verfügbar unter https://www.kea-bw.de/ fileadmin/user_upload/Publikationen/Experience_with_heat_planning_in_ Denmark_-_GermanSummary.pdf, zuletzt geprüft am 13.08.2020.

Hirsch-Kreinsen, Hartmut (2018): Das Konzept des Soziotechnischen Systems. Revisited. In: AlS-Studien 11 (2), S. 11-28. https://doi.org/10.21241/ssoar.64859 Kropp, Cordula (2017): Climate Change Governance. Möglichkeiten und Grenzen kollektiver Problemlösungsprozesse „von unten“. In: Jana Rückert-John und Martina Schäfer (Hg.): Governance für eine Gesellschaftstransformation. Wiesbaden: Springer Fachmedien Wiesbaden, S. 133-160. https://doi. org/10.1007/978-3-658-16560-4_6

Löw, Martina; Sept, Ariane (2019): Innovationen in Stadt und Raum. In: Birgit Blättel-Mink, Ingo Schulz-Schaeffer und Arnold Windeler (Hg.): Handbuch Innovationsforschung. Wiesbaden: Springer Fachmedien, S. 1-17. https://doi. org/10.1007/978-3-658-17671-6_49-1

Pehnt, Martin; Nast, Michael (2016): Wärmewende 2017. Impulse für eine klimafreundliche Wärmeversorgung. Online verfügbar unter https://www.boell.de/ sites/default/files/boellbrief_e-paper_waermewende.pdf, zuletzt geprüft am 13.08.2020.

Schieferdecker, Ina et al. (2018): Urbane Datenräume. Möglichkeiten von Datenaustausch und Zusammenarbeit im urbanen Raum. Berlin: Fraunhofer FOKUS.

Schmidt-Scheele, Ricarda et al. (2019): Leitmotive und Storylines der Energiewende. Wie Stakeholder-Positionen systematisch in Transformationspfade integriert werden können. In: TATuP - Zeitschrift für Technikfolgenabschätzung in Theorie und Praxis 28 (3), S. 27-33. https://doi.org/10.14512/ tatup.28.3.27

Sedlacek, Sabine; Tötzer, Tanja; Lund-Durlacher, Dagmar (2020): Collaborative governance in energy regions. Experiences from an Austrian region. In: Journal of Cleaner Production 256. https://doi.org/10.1016/j.jclepro.2020.120256

Soike, Roman; Libbe, Jens (2018): Smart Cities in Deutschland. Eine Bestandsaufnahme. Berlin: Deutsches Institut für Urbanistik.

Vogel, Hans-Josef; Weißer, Karlheinz; Hartmann, Wolf (2018): Smart City. Digitalisierung in Stadt und Land. Herausforderungen und Handlungsfelder. Wiesbaden: Springer Gabler. https://doi.org/10.1007/978-3-658-19046-0

Zensus (2011): Zensusdatenbank des Zensus 2011. Online verfügbar unter https://ergebnisse.zensus2011.de/, zuletzt geprüft am 26.08.2020.

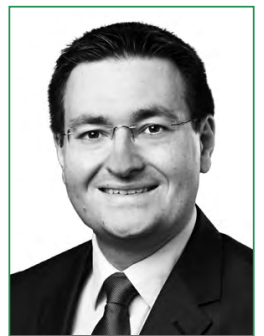

\section{DR.ANDRÉ ORTIZ}

ist Forschungskoordinator und Unternehmensberater bei der InWIS GmbH. Seine Arbeitsschwerpunkte liegen u. a. auf den Gebieten Innovationsmanagement, Digitalisierung und Smart City.

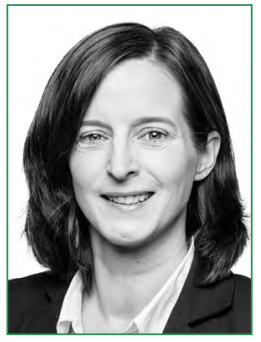

\section{DR. DOROTHEA LUDWIG}

leitet bei der IP SYSCON GmbH den Themenbereich Energie und Klima. Sie beschäftigt sich mit räumlichen Fragestellungen zur Energiewende und Klimaanpassung und entwickelt dafür Analysemethoden und WebGIS Anwendungen.

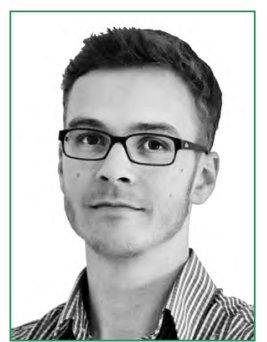

\section{FRITHJOF POLLMÜLLER}

ist bei der IP SYSCON GmbH als technischer Berater und Webentwickler im Bereich Energie und Klima tätig.

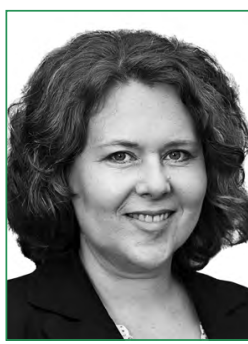

\section{DANIELA BECKER}

ist wissenschaftliche Mitarbeiterin im Arbeitsfeld Umweltpsychologie bei der IZES gGmbH, u. a. zu Themen der Beteiligung und des transformativen Prozess-Design. Dabei arbeitet sie in inter- und transdisziplinären Energiewende-Projekten, auch auf kommunaler Ebene. Sie ist zudem freiberufliche Prozessbegleiterin.

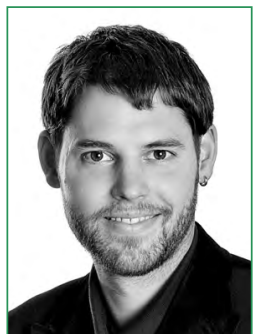

\section{FLORIAN NOLL}

ist seit 2008 wissenschaftlicher Mitarbeiter der IZES $\mathrm{gGmbH}$. Er beschäftigt sich hauptsächlich mit Entscheidungsprozessen rund um die kommunale Energiewende und wie diese durch digitale Hilfsmittel, wie das Wärmekataster, unterstützt werden können. 